\title{
SOBRE LAS DIFICULTADES DE ENTENDIMIENTO Y APRENDIZAJE DE LOS VERBOS DE MOVIMIENTO POLACOS
}

\begin{abstract}
Resumen. El objetivo del siguiente trabajo se centra en presentar las dificultades más destacables que tienen los hispanohablantes a la hora de entender y aprender los verbos de movimiento polacos. En primer lugar, se explican las diferencias generales entre el verbo español ir y sus equivalentes polacos. Posteriormente, se analizan los equivalentes polacos usando como base el concepto de la habitualidad y la direccionalidad. Una vez explicadas estas diferencias, se abordará el tema del aspecto de los verbos polacos y se presentarán los prefijos que se combinan con los verbos de movimiento modificado, tanto en su aspecto como en su significado.
\end{abstract}

Palabras clave: verbos de movimiento, verbos de desplazamiento, habitualidad, 97 direccionalidad, aspecto.

\section{Una introducción muy personal}

He madurado durante un largo tiempo la elección del tema para el artículo que se incorporará a un volumen publicado en homenaje a una persona tan especial como, sin duda alguna, es el profesor Wiaczesław Nowikow. He tenido que llevar a cabo una profunda reflexión en cuanto a todas las situaciones vivenciales y académicas en las que hemos tenido oportunidad de coincidir para poder centrar diversos aspectos que afloran en mi memoria. Recuerdo muy bien que nos conocimos en el Simposio Internacional titulado "Retos del Hispanismo en la Europa Central y del Este" organizado en octubre de 2005 en Cracovia, pero su nombre en aquel momento no me era ajeno, ya que como estudiante aprendía la fonética española apoyándome en su manual. Con posterioridad coincidimos en todos los simposios organizados por la Sociedad Polaca de Hispanistas, denominados "Encuentros", en jornadas organizadas

* Uniwersytet Śląski w Katowicach. 
por el Instituto Cervantes de Cracovia, y, por supuesto, en los "Foros" o congresos de lingüistas hispánicos polacos, que fueron auspiciados por el Profesor Nowikow. Debo subrayar que su idea de organizar los "Foros" cada dos años en diferentes centros académicos para conocernos mejor, tanto a nivel profesional como personal, fue y sigue siendo extraordinaria. Gracias a esta iniciativa todos los hispanistas que nos dedicamos al estudio de la lingüística en Polonia continuamos en contacto, intercambiando ideas y opiniones, a la par que colaboramos en distintas iniciativas, de modo que el hispanismo polaco va creciendo paulatinamente.

Puedo afirmar que nuestros caminos se cruzan constantemente y el profesor Nowikow siempre ha estado y sigue estando muy presente en mi vida profesional. Sus artículos y libros me sirven para desarrollar continuamente el conocimiento de la lengua española, a la par que sus ponencias me inspiran en la búsqueda de nuevos campos de investigación. Al mismo tiempo, durante estos años he tenido el placer de conocerlo también a nivel personal, por lo que puedo destacar que es una persona bondadosa y con un gran calado moral. Acompañado constantemente por una tenue sonrisa, un tanto callado, siempre disfruta enormemente de su trabajo y aún hoy se encuentra con fuerzas y ganas de ayudar a jóvenes hispanistas que empiezan su largo camino.

Además, dado que el Profesor Nowikow fue también el evaluador de mi tesis doctoral, dedicada al estudio contrastivo de los verbos de movimiento españoles y polacos, he decidido proseguir este rumbo $\mathrm{y}$, precisamente, porque me apasiona la enseñanza, poner en práctica lo que investigo aplicando la lingüística a la didáctica, por lo que he decidido ampliar este tema, aunque esta vez quiero abordarlo desde otra perspectiva: concretamente desde el punto de vista de la enseñanza del polaco a los hispanohablantes.

Antes de proseguir, deseo aprovechar la ocasión y expresar mi profunda alegría por tener la suerte de haber conocido en mi camino profesional al profesor Nowikow y darle las gracias por su amabilidad, por su sonrisa, por sus enseñanzas, por su ánimo y por su continua inspiración. 


\section{El desplazamiento}

Como bien es sabido, el polaco pertenece a la familia de las lenguas eslavas mientras que el español se adscribe a la familia de las lenguas románicas, estableciéndose una diferenciación en todos los niveles. En nuestro trabajo nos gustaría poner de manifiesto las diferencias más destacables relacionadas con la conceptualización del movimiento. Nuestra pretensión se centra en el objeto de ayudar a los hispanohablantes a acercarse a las distintas formas de describir el movimiento que tiene el polaco y a intentar a explicar el funcionamiento de los verbos de movimiento en esta lengua. Por lo tanto, no consiste nuestra intención en hacer un estudio lingüístico amplio y exhaustivo, sino abordar el tema desde el punto de vista didáctico, enumerando las diferencias más reseñables entre ambas lenguas, sin entrar en una descripción excesivamente detallada de todos los posibles usos de los verbos de movimiento.

Podemos comenzar argumentando que ambas lenguas poseen un verbo que describe de una forma muy general el movimiento más típico del ser humano y de muchos animales, es decir, el verbo desplazarse y su equivalencia polaca przemieszczać się, aunque se trata de verbos poco empleados.

Si queremos analizar los verbos que encabezan las primeras posiciones de las listas de frecuencia, debemos señalar al verbo $i r$, y a sus equivalentes polacos, cuestión que trataremos en los pasos siguientes.

\section{En busca de los equivalentes polacos del verbo ir}

Antes que nada, es necesario realizar una aproximación al significado del verbo español ir. Se suele usar fundamentalmente para hablar del desplazamiento ${ }^{1}$. Desde el punto de vista de nuestro análisis contrastivo polaco-español es muy importante apuntar que el verbo ir no incluye el modo de movimiento, dado que nos informa solamente sobre un desplazamiento ${ }^{2}$. Precisamente esta es la dificultad que encontramos a la hora de buscar un equivalente polaco, ya que los verbos polacos de

\footnotetext{
1 Aunque se suele usar también en las perífrasis verbales tipo: $i r+a$, ir + gerundio.

${ }^{2}$ Los primeros investigadores que se enfocaron en los diferentes modos de expresar el movimiento en varias lenguas fueron Vinay y Darbelnet (1958), después podemos destacar estudios hechos por Talmy $(1985,1991)$ y Slobin (1996).
} 
movimiento incluyen el modo de desplazamiento ${ }^{3}$. Por este motivo, para expresar lo que señala el verbo ir debemos recurrir a distintos verbos en polaco. El primero de ellos es iść, que expresa el desplazamiento a pie, es decir, ir andando, o jechać, que significa trasladarse en un medio de transporte sin especificar. Estos dos verbos los trataremos como equivalentes más cercanos al verbo ir; sin embargo, cabe subrayar que entre otros equivalentes podemos encontrar también el verbo lecieć que significa ir volando, płynać, ir nadando o biec, ir corriendo.

Como podemos observar, la lengua española expresa el modo de movimiento añadiendo al verbo ir otro verbo en gerundio. Pongamos, por ejemplo, ir andando, -de acuerdo con la división hecha por Morimoto (2001)-: el verbo ir pertenece (junto con salir, subir, bajar, cruzar, entrar, etc.) al grupo de los Verbos de Desplazamiento (VVDD), que incluye la propiedad de la direccionalidad, a diferencia de lo que ocurre con el grupo de los Verbos de Movimiento (VMMs) del tipo caminar (andar, correr, nadar, volar, etc.). Esto quiere decir que en español empleamos el primer grupo para expresar un desplazamiento con una determinada dirección, mientras que en el segundo, lo hacemos para señalar la existencia de un desplazamiento. De igual modo, se pueden unir los dos grupos expresando, al mismo tiempo, tanto la direccionalidad como el modo de movimiento. Sin embargo, en polaco los verbos iść, jechać, lecieć, ptynać o biec incluyen el modo, por lo que expresan tanto la dirección como el modo de movimiento. Veamos algunos ejemplos:

1. Ide do szkoty [Voy andando a la escuela] $]^{4}$.

2. Jadę do pracy [Voy al trabajo (en un medio de transporte)].

3. Lecę do Ameryki [Voy en avión a América].

4. Ptynę do Grecji [Voy en barco a Grecia].

5. Biegne do domu [Voy corriendo a casa].

Cabe añadir, que en muchas ocasiones el verbo jechać sustituye a los verbos lecieć y płynać, ya que expresa el desplazamiento sin indicar el modo de transporte. Así pues, en las frases 3 y 4 el verbo expresado se puede sustituir perfectamente por el verbo jechać (Jadę do Ameryki. Jadę do Grecji.), aunque en este caso se supondría que el hablante usa el avión o el barco como medio de transporte, según el contexto.

${ }^{3}$ Siguiendo a Talmy $(1985,1991)$, tendríamos que indicar que el español se inscribe en el grupo de lenguas de enmarcamiento verbal, mientras que el polaco lo hace en el grupo de enmarcamiento por satélite.

${ }^{4}$ N. de la Aut.: entre corchetes ofrecemos las traducciones lo más cercanas posible al original con el objetivo de transmitir toda la información que incluye la versión polaca. 


\section{La habitualidad y la direccionalidad}

Para comprender mejor la conceptualización de los verbos de movimiento polacos, hay que añadir que dichos verbos aparecen emparejados. Por ello, junto a los que acabamos de presentar (iść, jechać, lecieć, płynać, biec) tenemos que añadir los siguientes: chodzić, jeździć, latać, pływać y biegać. Las parejas se diferencian por una de las dos características que denominamos ‘habitualidad' y 'direccionalidad'. Así pues, los verbos iść, jechać, lecieć, płynać, se utilizan para hablar de un movimiento que se realiza una sola vez, mientras que los segundos sirven para hablar de un desplazamiento habitual. De este modo, podríamos transformar las frases que hemos visto con anterioridad en las siguientes:

6. Chodze do szkoty [Voy andando a la escuela con regularidad].

7. Jeżdżę do pracy [Voy al trabajo (en un medio de transporte) con regularidad].

8. Latam do Ameryki [Voy en avión a América con regularidad].

9. Ptywam do Grecji [Voy en barco a Grecia con regularidad].

10. Biegam do domu [Voy corriendo a casa con regularidad].

Esta es una manera de diferenciar las parejas de verbos, aunque, por otro lado, la segunda forma consistiría en atender a la otra característica, es decir, a la direccionalidad. Esto quiere decir que los verbos iść, jechać, lecieć, płynać o biec expresan un desplazamiento con una dirección concreta, mientras que los verbos chodzić, jeździć, latać, ptywać y biegać, o bien expresan un movimiento sin ninguna dirección o bien un movimiento habitual (tal como hemos podido apreciar con anterioridad) con una dirección o con varias direcciones concretas. Dicho esto, procedemos a ofrecer ejemplos del primer caso en las siguientes oraciones:

11. Chodze po parku [Ando por el parque].

12. Jeżdże po ulicy [Circulo por la calle (en un medio de transporte)].

13. Latam po niebie [Vuelo por el cielo].

14. Pływam w rzece [Nado en el río].

15. Biegam po mieście [Corro por la ciudad].

Todos estos verbos describen un movimiento sin una dirección concreta. En este caso se aproximan bastante a los verbos españoles que Morimoto (2001) agrupa bajo el nombre de Verbos de Movimiento (VMMs) del tipo de caminar (andar, correr, nadar, volar, etc.).

Al mismo tiempo, los verbos chodzić, jeździć, latać, pływać y biegać pueden expresar un movimiento habitual en una dirección (hecho ya mencionado) o en varias direcciones, como por ejemplo: 
16. Chodze do różnych sklepów [Voy regularmente a varias tiendas].

17. Jeżdże do różnych supermarketów [Voy regularmente (en un medio de transporte) a varios almacenes].

18. Latam do Stanów $i$ do Hiszpanii [Vuelo regularmente a los EEUU y a España].

19. Ptywam do Grecji i do Egiptu [Voy en barco regularmente a Grecia y a Egipto].

20. Biegam do sklepu i do domu [Voy corriendo regularmente a la tienda y a casa].

\section{El aspecto, los prefijos y el cambio del significado}

La parte más complicada para entender la conceptualización del movimiento para aquellos que quieran aprender la lengua polaca es, sin duda alguna, el tema del aspecto, relacionado con los prefijos que influyen significativamente en el cambio del significado.

No resulta nada fácil hacer un análisis contrastivo entre ambas lenguas, ya que, como señala Nowikow (2012: 114): «Las diferencias básicas entre el castellano y el polaco son de índole tipológica». Tal y como explica el autor, se debe a que (aunque no solamente por eso) en la lengua polaca el aspecto gramatical desempeña un papel determinante, mientras que en el español lo hace el llamado aktionsart, es decir, el modo accional. Sin entrar en descripciones detalladas del tema ${ }^{5}$, en este apartado nos enfocaremos sobre todo en la lengua polaca, sin entrar en la determinación del aspecto de los verbos españoles.

Un inicio del tratamiento que se otorga al verbo parte de las tesis de Nowikow (2012: 101), quien dice que:

[...] casi todos los verbos polacos o rusos poseen pares aspectuales ya a nivel de infinitivo (igual que, p. ej., los sustantivos que se caracterizan por el género) sin que exista la conjugación aspectual propiamente dicha. Además, el medio más habitual para la creación de las formas aspectuales es el de la formación de las unidades léxicas, o sea, la derivación mediante prefijos:

aspecto imperfectivo

pol. publikować

pol. jeść

pol. kończyć

o sufijos:

aspecto imperfectivo

pol. wstawać

pol. odwiedzać

pol. wracać aspecto perfectivo

opublikować esp. publicar zjeść esp. comer skończyć esp. terminar

\footnotetext{
aspecto perfectivo wstać esp. levantarse odwiedzić esp. visitar wrócić esp. regresar.
}

${ }^{5}$ A las personas interesadas en el tema del aspecto les recomendamos una revisión de los trabajos del profesor Nowikow que se mencionan en la bibliografía. 
Los verbos de movimiento que hemos mostrado anteriormente, tanto los que expresan direccionalidad (iść, jechać, lecieć, płynać, biec), como los que expresan la falta de direccionalidad o habitualidad (chodzić, jeździć, latać, pływać, biegać), son verbos con aspecto imperfectivo, dicho de otra forma: son predicados dinámicos de acción, que se basan en el concepto simple de CONTINUIDAD. Como subraya Wróbel (1998: 555), estos verbos de movimiento direccionalizado expresan un desplazamiento de un objeto en una dirección, pero sin determinar con precisión los puntos que recorren en el espacio. Son los prefijos los que ofrecen esta información adicional, pero hay que entender que dichos verbos no tienen formas homólogas perfectivas porque hay que tener en cuenta que «los prefijos polacos, además de ser marcadores aspectuales (en este caso de la perfectividad), desempeñan otras funciones semánticas, que pueden llegar a ser sus exponentes principales» (Wilk-Racięska, 2004). Por tanto, los prefijos que se anteponen a los verbos iść o jechać, indican la direccionalidad y al mismo tiempo expresan la perfectividad. De ahí deducimos que los verbos przyjść, odejść, etc. [venir / llegar, irse] forman parejas con iść, jechać, pero no son sus equivalentes 'puros' ${ }^{6}$.

Por añadidura, aunque consideramos perfectivos los verbos de movimiento con prefijos, vemos que estos no expresan un resultado entendido como «un cambio intrínseco que afecte al sujeto» (WilkRacięska, 2004: 64). No obstante, según Wilk-Racięska (2004: 64), ello no significa que los semantemas que representan aquellos predicados, no puedan expresar tal estado resultante de alguna manera. Siendo discontinuos, aquellos predicados encierran en su estructura el concepto de LÍMITE.

Todos los verbos de movimiento que estamos analizando pueden ir acompañados de prefijos que, al mismo tiempo, cambian el aspecto del verbo y, por ende, su significado. Para simplificar un poco la presente descripción, no nos centraremos solamente en los cuatro verbos que más se acercan al significado del verbo español $i r$, es decir, a los verbos anteriormente mencionados iść, jechać, chodzić y jeździć. El verbo que más sufijos acepta es el verbo iść, al que se añaden 15 sufijos, formando los siguientes verbos perfectivos: przyjść, dojşć, podejść, nadejść, zajść, pójssć, wejść, odejść, rozejść, zejść, ujść, wyjsść, wzejść, przejść y przechodzić. Cabe subrayar que los mismos prefijos (desde el punto de vista de la fonología), pueden añadir diferentes significados. Por ejemplo, el prefijo pod- unido al verbo iść (esp. ir) (podejść, podchodzić) significa acercarse, pero también puede describir un desplazamiento desde abajo.

\footnotetext{
${ }^{6}$ Véase también Wróbel (1998: 543-544).
} 
Por su parte, Wróbel (1998: 555-557) divide los prefijos en tres grupos generales: los que indican el acercamiento, los que indican el adelantamiento y otros que incluyen otras informaciones en cuanto a la direccionalidad. Por ejemplo, en el primer grupo encontramos los siguientes prefijos: $p r z y-$, pod-, nad-, $z a-$, po-, do-, que se suelen traducir con los verbos venir, llegar o acercarse ${ }^{7}$.

\section{Conclusiones}

El objetivo de nuestro trabajo se ha centrado en mostrar las diferencias más destacables entre la conceptualización del movimiento en español y en polaco. Hemos puesto de manifiesto que, aunque describimos las mismas situaciones que son típicas a todas las personas del mundo, existen en polaco diferentes aspectos en los que focalizarse. Desde el punto de vista de la lengua polaca es importante, por un lado, el modo de desplazamiento, especialmente si se trata de indicar si se hace andando o en un medio de transporte, aunque por otro, también es importante resaltar si es un movimiento habitual o puntual; otra cuestión adyacente, como hemos visto, se focaliza en si tiene una dirección concreta o no. Además, a los verbos de desplazamiento se suelen añadir prefijos que modifican su aspecto y añaden informaciones acerca de la direccionalidad. Al mismo tiempo, entendemos que el tema abordado es solamente una introducción a una cuestión mucho más amplia que necesitaría un estudio en profundidad.

\section{Bibliografía}

MORIMOTO, Y. (2001). Los verbos de movimiento. Madrid: Visor Libros.

NOWIKOW, W. (2010a). «Algunas observaciones sobre los aspectos metodológicos del estudio contrastivo de los sistemas verbales del español y del polaco", Kwartalnik Neofilologiczny, LVII, 2, 131-138.

NOWIKOW, W. (2010b). «En torno a la interferencia en la enseñanza de los pretéritos a los alumnos polacos: tiempo vs. Aspecto», en G. LUQUET y W. NOWIKOW (eds.), La recherche en langues romanes. Théories et applications. Łódź: Lodz Academie of International Studies / Université Sorbonne Nouvelle-Paris, 3, 289-302.

${ }^{7}$ Para obtener una explicación exacta de los formantes con ejemplos y su traducción al español, consúltese Tatoj (2012a). 
NOWIKOW, W. (2012). «La organización de los sistemas temporales del español y del polaco: análisis contrastivo de los tiempos verbales», Studia Iberystyczne, 11, 119-134.

SLOBIN, D. I. (1996). «Two ways to travel: verbs of motion in English and Spanish», en M. SHIBATANI y S. A. THOMPSON (eds.), Grammatical Constructions: Their Form and Meaning. New York: Oxford University Press, 195-219.

TALMY, L. (1985). «Lexicalization patterns: semantic structure in lexical forms», en T. SHOPEN (ed.), Language typology and syntactic description. Cambridge: Cambridge University Press, vol. 3, 36-149.

TALMY, L. (1991). «Path to realization: a typology of event conflation», Berkeley Linguistic Society, 7, 480-519.

TATOJ, C. (2010). «¿Qué escenas describe el verbo español ir y sus equivalentes polacos? Análisis contrastivo», Verba Hispanica. Anuario del Departamento de Lengua y Literatura españolas de la Facultad de Filosofía y Letras de la Universidad de Ljubljana, vol. XVIII, 161-173.

TATOJ, C. (2010). «Wyrażanie przemieszczania się w języku hiszpańskim i polskim. Najważniejsze różnice na podstawie analizy wybranych czasowników», en M. MICHALSKA-SUCHANEK (ed.), Literatura i język. Szkice opisowe i komparatywne. Prace Naukowe GWSP. Gliwice: Gliwicka Wyższa Szkoła Przedsiębiorczości, 124-131.

TATOJ, C. (2012a). «El tiempo y el espacio: análisis del aspecto de unos verbos de movimiento españoles y polacos escogidos», Verba Hispanica. Anuario del Departamento de la Lengua y Literatura españolas de la Facultad de Filosofía y Letras de la Universidad de Ljubljana, vol. XX, 345-362.

TATOJ, C. (2012b). «Análisis del significado de los verbos españoles venir y llegar, y sus equivalentes polacos», Neophilologica, 24, 240-252.

TATOJ, C. (2014). «Análisis de errores de interferencia en la descripción del movimiento en la clase de traducción español-polaco», en M. ŚWIĄTEK (ed.), Enseñanza y aprendizaje del idioma español. Retos, propuestas y perspectivas. Wrocław: Wydawnictwo Uniwersytetu Wrocławskiego, 49-58.

VINAY, J. P. y DARBELNET, J. (1958). Stylistique comparée du français et de l'anglais. París: Didier.

WILK-RACIĘSKA, J. (2004). El tiempo interior. Una aproximación al aspecto en español. Katowice: Wydawnictwo Uniwersytetu Śląskiego.

WILK-RACIĘSKA, J. (2006). «¿Es posible que un español aprenda a expresar correctamente el aspecto en polaco? Una opinión más sobre la naturaleza del aspecto», Mundo Eslavo, 5, 141-152.

WRÓBEL, H. (1979). «Współczesne i staropolskie czasowniki z przedrostkiem do-», en J. SAFAREWICZ et al. (eds.), Opuscula Polono-Slavica. Wrocław, Warszawa, Kraków: Zakład Narodowy im. Ossolińskich, 415-421.

WRÓBEL, H. (1998). «Czasownik», en R. GRZEGORCZYKOWA, R. LASKOWSKI y H. WRÓBEL (eds.), Gramatyka wspótczesnego języka polskiego. Warszawa: PWN, 536-568. 